\title{
Special issue: The International Conference on Soft Computing and Machine Intelligence
}

\author{
Suash Deb ${ }^{1,2} \cdot$ Thomas Hanne $^{3} \cdot$ Xiao-Zhi Gao $^{4}$ \\ Published online: 25 June 2018 \\ (C) The Natural Computing Applications Forum 2018
}

It is a privilege for us to present this Special Issue (SI) of the journal "Neural Computing \& Applications" as guest editors and write the editorial. Before introducing the papers that appear in this SI, let us briefly throw some light on ISCMI15.

The field of soft computing has evolved rapidly over the years which results in the development of new methodologies and techniques that enhance, among others, its application areas. The International Conference on Soft Computing and Machine Intelligence (ISCMI) has established itself as a broad interdisciplinary forum for academics, researchers, and practitioners who are involved in the investigation and development of robust intelligent computing to overcome a number of limitations that are even associated with high-performance conventional computing. ISCMI15 was the second in this series, which took place in Hong Kong from 23 to 24 November, 2015.

The six papers included in this special issue represent different areas where theory meets engineering practice. These six papers are a very small subset of all manuscripts accepted and presented at ISCMI15. All the papers presented, as they appeared in the proceedings of ISCMI15, were carefully examined by a group of experts, which resulted in the shortlisting of 15 papers. The authors of these papers were advised to expand their respective

Suash Deb

suashdeb@gmail.com

Thomas Hanne

thomas.hanne@fhnw.ch

Xiao-Zhi Gao

xiao.z.gao@gmail.com

1 IT \& Educational Consultant, Ranchi, Jharkhand, India

2 Decision Sciences and Modelling Program, Victoria University, Melbourne, Australia

3 University of Applied Sciences and Arts Northwestern Switzerland, Olten, Switzerland

4 University of Eastern Finland, Kuopio, Finland manuscripts by at least thirty percent and submit them for review, using the same procedure as for every regular paper submitted to the NCAA. Based on the reviewers' input, six papers were finally selected for publication in this SI.

Although we aimed at a SI that offers a fair representation of three methodologies, i.e. artificial neural networks, fuzzy logic and evolutionary computation, it turned out that the majority of the finally accepted papers deal with metaheuristics/evolutionary computation. In fact, with the exception of the second paper which presents the development of a new neuro-fuzzy inference system with application to the areas of power conditioning, all other papers fall into this category.

The first paper represents the application of the particle swarm optimization methodology for addressing the identification of infinite impulse response systems. The third paper investigates the possible parallelization of the efficient distribution of a multi-objective optimization task to various devices with the aim of reducing the computational time to a large extent. The next paper emphasizes on the fact that knowledge, in various forms, can promote more efficient evolutionary algorithms and presents multi-objective quantum-inspired cultural algorithms, which can harness implicit information, provided in evolution, to promote a more efficient search. The fifth article reports the development of a new symmetry-based clustering algorithm by utilizing a fuzzy point symmetric concept along with the search capability of the particle swarm optimization methodology and the utility of the resultant fuzzy-PSO clustering which was demonstrated to partition gene expression datasets into a number of clusters, which was not pre-specified. This SI ends with the manuscript which introduces and validates a novel recombination operator for a multi-objective evolutionary algorithm, called hybrid crossover operator.

We hope that this SI will lay a foundation for the researchers to focus on possible future developments and that it will inspire more research that will open new directions in relation to the field of soft computing. We are 
grateful to Prof. John MacIntyre, the editor-in-chief of this journal for enabling us to guest edit this SI as well as for his guidance throughout this work. We also wish to acknowledge the help provided by the editorial office and especially that of Ms. Ramya Balu. The reviewers, despite their very busy schedule, did a great job of sending their valuable review inputs on time. Before ending, we would like to thank the authors for showing great patience during the review process of their manuscripts. 